\title{
SCHATTEN CLASS POSITIVE TOEPLITZ OPERATORS ON BERGMAN SPACES OF THE SIEGEL UPPER HALF-SPACE
}

\author{
JIAJIA SI
}

\begin{abstract}
We characterize Schatten class membership of positive Toeplitz operators defined on the Bergman spaces over the Siegel upper half-space in terms of averaging functions and Berezin transforms in the range of $0<p<\infty$.
\end{abstract}

\section{INTRODUCTION}

Let $\mathbb{C}^{n}$ be the $n$-dimensional complex Euclidean space. For any two points $z=\left(z_{1}, \cdots, z_{n}\right)$ and $w=\left(w_{1}, \cdots, w_{n}\right)$ in $\mathbb{C}^{n}$ we write

$$
z \cdot \bar{w}:=z_{1} \bar{w}_{1}+\cdots+z_{n} \bar{w}_{n}
$$

and

$$
|z|:=\sqrt{z \cdot \bar{z}}=\sqrt{\left|z_{1}\right|^{2}+\cdots+\left|z_{n}\right|^{2}} .
$$

The set

$$
\mathcal{U}=\left\{z \in \mathbb{C}^{n}: \operatorname{Im} z_{n}>\left|z^{\prime}\right|^{2}\right\}
$$

is the Siegel upper half-space. Here and throughout the paper, we use the notation

$$
z=\left(z^{\prime}, z_{n}\right), \text { where } z^{\prime}=\left(z_{1}, \cdots, z_{n-1}\right) \in \mathbb{C}^{n-1} \text { and } z_{n} \in \mathbb{C}^{1} .
$$

The Bergman space $A^{2}(\mathcal{U})$ is the space of all complex-valued holomorphic functions $f$ on $\mathcal{U}$ such that

$$
\int_{\mathcal{U}}|f|^{2} d V<\infty
$$

where $V$ denotes the Lebesgue measure on $\mathcal{U}$. It is a closed subspace of $L^{2}(\mathcal{U})$ and hence a Hilbert space. The orthogonal projection from $L^{2}(\mathcal{U})$ onto $A^{2}(\mathcal{U})$ can be expressed as an integral operator:

$$
\operatorname{Pf}(z)=\int_{\mathcal{U}} K(z, w) f(w) d V(w),
$$

with the Bergman kernel

$$
K(z, w)=\frac{n !}{4 \pi^{n}}\left[\frac{i}{2}\left(\bar{w}_{n}-z_{n}\right)-z^{\prime} \cdot \bar{w}^{\prime}\right]^{-n-1} .
$$

See, for instance, [4, Theorem 5.1]. The operator $P$ is usually called a Bergman projection. It is a bounded projection from $L^{2}(\mathcal{U})$ onto $A^{2}(\mathcal{U})$, see [3] Lemma 2.8].

2010 Mathematics Subject Classification. Primary 47B35; Secondary 32A36.

Key words and phrases. Toeplitz operators; Schatten classes; Bergman spaces; Siegel upper half-space; Berezin transform.

This work was supported by the National Natural Science Foundation of China grant 11971453. 
Let $\mathcal{M}_{+}$be the set of all positive Borel measures $\mu$ such that

$$
\int_{\mathcal{U}} \frac{d \mu(z)}{\left|z_{n}+i\right|^{\alpha}}<\infty
$$

for some $\alpha>0$. Given $\mu \in \mathcal{M}_{+}$, the Toeplitz operator $T_{\mu}$ with symbol $\mu$ is given by

$$
T_{\mu} f(z)=\int_{\mathcal{U}} K(z, w) f(w) d \mu(w)
$$

for $f \in A^{2}(\mathcal{U})$. In case $d \mu=g d V$, we write $T_{\mu}=T_{g}$. In general, $T_{\mu}$ may not even be defined on all of $A^{2}(\mathcal{U})$, but it is always densely defined by the fact that, for each $\alpha>n+1 / 2$, holomorphic functions $f$ on $\mathcal{U}$ such that $f(z)=O\left(\left|z_{n}+i\right|^{-\alpha}\right)$ form a dense subset of $A^{2}(\mathcal{U})$, see [7].

For a positive Borel measure $\mu$ on $\mathcal{U}$, the Berezin transform of $\mu$ is given by

$$
\widetilde{\mu}(z):=\int_{\mathcal{U}}\left|k_{z}(w)\right|^{2} d \mu(w), \quad z \in \mathcal{U}
$$

where

$$
k_{z}(w):=K(z, w) / \sqrt{K(z, z)}, \quad w \in \mathcal{U} .
$$

For $z \in \mathcal{U}$ and $r>0$, we define the averaging function

$$
\widehat{\mu}_{r}(z):=\frac{\mu(D(z, r))}{|D(z, r)|},
$$

where $D(z, r)$ is the Bergman metric ball at $z$ with radius $r$ and $|D(z, r)|$ denotes the Lebesgue measure of $D(z, r)$.

Quite recently in [7, we have characterized the boundedness and compactness of $T_{\mu}$ on Bergman spaces in terms of $\widetilde{\mu}$ and $\widehat{\mu}_{r}$. In the paper, we are going to study the membership of $T_{\mu}$ in Schatten class $S_{p}$ in terms of $\widetilde{\mu}$ and $\widehat{\mu}_{r}$. It is a natural subsequent work of [7]. Our main result is the following.

Theorem 1.1. Suppose $0<p<\infty, r>0$ and $\mu \in \mathcal{M}_{+}$. Then the following conditions are equivalent:

(i) $T_{\mu} \in S_{p}$.

(ii) $\widehat{\mu}_{r} \in L^{p}(\mathcal{U}, d \lambda)$.

(iii) $\left\{\widehat{\mu}_{r}\left(a_{k}\right)\right\} \in l^{p}$ for every $r$-lattice $\left\{a_{k}\right\}$.

(iv) $\left\{\widehat{\mu}_{r}\left(a_{k}\right)\right\} \in l^{p}$ for some r-lattice $\left\{a_{k}\right\}$.

Moreover, if $p>n /(n+1)$, then the above conditions are also equivalent to

(v) $\tilde{\mu} \in L^{p}(\mathcal{U}, d \lambda)$.

Here $d \lambda(z)=K(z, z) d V(z)$ is the Möbius invariant measure on $\mathcal{U}$ (see [5, Proposition 1.4.12] for example) and the cut-off point $n /(n+1)$ is sharp.

The equivalences of (i), (iii) and (iv) were originally proved by Luecking 8 for the full range $0<p<\infty$ in the case of the unit disk. Later Zhu [10] extended Luecking's result to bounded symmetric domains and added condition (v), with the restricted range $1 \leq p<\infty$. After that, Zhu [11 continued to study similar characterizations for the range $0<p<1$ on the unit ball. His result reveals an interesting aspect that, the full range $0<p<1$ for characterization with averaging function is appropriate, while this is not the case for characterization with Berezin transform. On the setting of harmonic Bergman space of the upper half-space of $\mathbb{R}^{n}$, Choe et al. obtained similar characterizations, see [1, 2]. 
The paper is organized as follows. In Section 2 we collect some basic auxiliary results. Section 3 is devoted to show the equivalences of (ii)-(iv). In Section 4 we prove Theorem 1.1 in the case of $p \geq 1$, where also we recall the notion and some elementary facts of Schatten class operators. Section 5 is devoted to show the equivalences of (i)-(iv) in the range of $0<p<1$. The characterization of Schatten class Toeplitz operators in terms of the Berezin transform is obtained in Section 6 .

Throughout the paper, the letter $\mathrm{C}$ will denote a positive constant that may vary at each occurrence but is independent of the essential variables.

\section{Preliminaries}

In this section we introduce notations and collect several basic lemmas which will be used in later sections. Throughout the paper, we write

$$
\boldsymbol{\rho}(z, w):=\frac{i}{2}\left(\bar{w}_{n}-z_{n}\right)-z^{\prime} \cdot \overline{w^{\prime}}
$$

and let $\boldsymbol{\rho}(z):=\boldsymbol{\rho}(z, z)=\operatorname{Im} z_{n}-\left|z^{\prime}\right|^{2}$ for simplicity. With this notation, the Bergman kernel of $\mathcal{U}$ is

$$
K(z, w)=\frac{n !}{4 \pi^{n}} \frac{1}{\boldsymbol{\rho}(z, w)^{n+1}}, \quad z, w \in \mathcal{U} .
$$

For each $t>0$, we define the nonisotropic dilation $\delta_{t}$ by

$$
\delta_{t}(u)=\left(t u^{\prime}, t^{2} u_{n}\right), \quad u \in \mathcal{U}
$$

Also, to each fixed $z \in \mathcal{U}$, we associate the following (holomorphic) affine selfmapping of $\mathcal{U}$ :

$$
h_{z}(u):=\left(u^{\prime}-z^{\prime}, u_{n}-\operatorname{Re} z_{n}-2 i u^{\prime} \cdot \overline{z^{\prime}}+i\left|z^{\prime}\right|^{2}\right), \quad u \in \mathcal{U} .
$$

All these mappings are holomorphic automorphisms of $\mathcal{U}$. See [9, Chapter XII]. Hence the mappings $\sigma_{z}:=\delta_{\boldsymbol{\rho}(z)^{-1 / 2}} \circ h_{z}$ are holomorphic automorphisms of $\mathcal{U}$. Simple calculations show that $\sigma_{z}(z)=\mathbf{i}:=\left(0^{\prime}, i\right)$.

Lemma 2.1. Suppose $z, u, v \in \mathcal{U}$, we have

$$
\begin{aligned}
\boldsymbol{\rho}\left(\sigma_{z}(u), \sigma_{z}(v)\right) & =\boldsymbol{\rho}(z)^{-1} \boldsymbol{\rho}(u, v), \\
\boldsymbol{\rho}\left(\sigma_{z}^{-1}(u), \sigma_{z}^{-1}(v)\right) & =\boldsymbol{\rho}(z) \boldsymbol{\rho}(u, v) .
\end{aligned}
$$

Proof. First note that

$$
\boldsymbol{\rho}\left(\delta_{t}(u), \delta(v)\right)=t^{2} \boldsymbol{\rho}(u, v) .
$$

Also, an easy calculation shows that

$$
\boldsymbol{\rho}\left(h_{z}(u), h_{z}(v)\right)=\boldsymbol{\rho}(u, v) .
$$

Then a combination of the two above equalities gives

$$
\begin{aligned}
\boldsymbol{\rho}\left(\sigma_{a}(z), \sigma_{a}(w)\right) & =\boldsymbol{\rho}\left(\delta_{\boldsymbol{\rho}(a)^{-1 / 2}}\left(h_{a}(z)\right), \delta_{\boldsymbol{\rho}(a)^{-1 / 2}}\left(h_{a}(w)\right)\right) \\
& =\boldsymbol{\rho}(a)^{-1} \boldsymbol{\rho}\left(h_{a}(z), h_{a}(w)\right) \\
& =\boldsymbol{\rho}(a)^{-1} \boldsymbol{\rho}(z, w),
\end{aligned}
$$

which is the first equality. The proof of the second one is exactly the same by observing that

$$
\boldsymbol{\rho}\left(h_{z}^{-1}(u), h_{z}^{-1}(v)\right)=\boldsymbol{\rho}(u, v)
$$

and

$$
\sigma_{z}^{-1}=h_{z}^{-1} \circ \delta_{\rho(z)^{1 / 2}}
$$


So we are done.

We recall from [7] that the Bergman metric on $\mathcal{U}$ is given by

$$
\beta(z, w)=\tanh ^{-1} \sqrt{1-\frac{\boldsymbol{\rho}(z) \boldsymbol{\rho}(w)}{|\boldsymbol{\rho}(z, w)|^{2}}},
$$

and the Bergman metric ball at $z$ with radius $r>0$ is denoted by

$$
D(z, r)=\{w \in \mathcal{U}: \beta(z, w)<r\} .
$$

A sequence $\left\{a_{k}\right\}$ in $\mathcal{U}$ is called an $r$-lattice in the Bergman metric, or an $r$-lattice for short, if it satisfies the following conditions:

(i) $\mathcal{U}=\bigcup_{k=1}^{\infty} D\left(a_{k}, r\right)$;

(ii) $\beta\left(a_{i}, a_{j}\right) \geq r / 2$ for all $i \neq j$.

For any $r>0$, the existence of an $r$-lattice can be verified by the proof of [12, Theorem 2.23]. Also, associated with a standard maximality argument, we have the following lemma.

Lemma 2.2. If $\left\{a_{k}\right\}$ is an r-lattice, then it has the following properties:

(i) For any $R>0$, there exists a positive integer $N$ (depending on $r$ and $R$ ) such that each point in $\mathcal{U}$ belongs to at most $N$ of the sets $\left\{D\left(a_{k}, R\right)\right\}$.

(ii) For any $R>0$, there is a finite partition $\left\{a_{k}\right\}=\Gamma_{1} \cup \cdots \cup \Gamma_{m}$ such that for every $i \in\{1, \cdots, m\}$, the conditions $u, v \in \Gamma_{i}$ and $u \neq v$ imply that $\beta(u, v)>R$

We need the following result [6, Lemma 5], which will be used in a few times.

Lemma 2.3. Let $s, t \in \mathbb{R}$. Then we have

$$
\int_{\mathcal{U}} \frac{\boldsymbol{\rho}(w)^{t}}{|\boldsymbol{\rho}(z, w)|^{s}} d V(w)= \begin{cases}\frac{C(n, s, t)}{\boldsymbol{\rho}(z)^{s-t-n-1},} & \text { if } t>-1 \text { and } s-t>n+1 \\ +\infty, & \text { otherwise }\end{cases}
$$

for all $z \in \mathcal{U}$, where

$$
C(n, s, t):=\frac{4 \pi^{n} \Gamma(1+t) \Gamma(s-t-n-1)}{\Gamma^{2}(s / 2)} .
$$

The following results can be found in [7, they serve as basic tools in this paper.

Lemma 2.4. We have

$$
2|\boldsymbol{\rho}(z, w)| \geq \max \{\boldsymbol{\rho}(z), \boldsymbol{\rho}(w)\}
$$

for any $z, w \in \mathcal{U}$.

Lemma 2.5. For any $z \in \mathcal{U}$ and $r>0$ we have

$$
|D(z, r)|=\frac{4 \pi^{n}}{n !} \frac{\tanh ^{2 n} r}{\left(1-\tanh ^{2} r\right)^{n+1}} \rho(z)^{n+1} .
$$

Consequently, the averaging function

$$
\widehat{\mu}_{r}(z)=\frac{n !}{4 \pi^{n}} \frac{\left(1-\tanh ^{2} r\right)^{n+1}}{\tanh ^{2 n} r} \frac{\mu(D(z, r))}{\boldsymbol{\rho}(z)^{n+1}} .
$$


Lemma 2.6. Given $r>0$, the inequalities

$$
\frac{1-\tanh (r)}{1+\tanh (r)} \leq \frac{|\boldsymbol{\rho}(z, u)|}{|\boldsymbol{\rho}(z, v)|} \leq \frac{1+\tanh (r)}{1-\tanh (r)}
$$

hold for all $z, u, v \in \mathcal{U}$ with $\beta(u, v) \leq r$.

Lemma 2.7. Suppose $r>0$ and $p>0$. Then there exists a positive constant $C$ depending on $r$ such that

$$
|f(z)|^{p} \leq \frac{C}{\boldsymbol{\rho}(z)^{n+1}} \int_{D(z, r)}|f(w)|^{p} d V(w)
$$

for all $f \in H(\mathcal{U})$ and all $z \in \mathcal{U}$.

The next lemma is a key result of [7, we shall use it without any explanation, since it is so natural and important.

Lemma 2.8. Let $\mu \in \mathcal{M}_{+}$and $T_{\mu}$ is bounded on $A^{2}(\mathcal{U})$. Then the equality

$$
\left\langle T_{\mu} f, g\right\rangle=\int_{\mathcal{U}} f \bar{g} d \mu
$$

holds for all $f, g \in A^{2}(\mathcal{U})$, where $\langle\cdot, \cdot\rangle$ denotes the inner product on $A^{2}(\mathcal{U})$.

\section{Averaging Functions}

In this section we characterize the $L^{p}(d \lambda)$-behavior of averaging functions as well as its discretized version. We begin with an observation from (2.4) and (2.6) that there is a positive constant $C$ depending only on $r$ such that

$$
\lambda(D(z, r)) \leq C
$$

for all $z \in \mathcal{U}$.

Lemma 3.1. Suppose $\mu \geq 0, r, \delta>0$ and $0<p<\infty$. If $\left\{\widehat{\mu}_{r}\left(a_{k}\right)\right\} \in l^{p}$ for some $r$-lattice $\left\{a_{k}\right\}$, then $\widehat{\mu}_{\delta} \in L^{p}(\mathcal{U}, d \lambda)$.

Proof. Assume that $\left\{a_{k}\right\}$ is an $r$-lattice such that $\left\{\widehat{\mu}_{r}\left(a_{k}\right)\right\} \in l^{p}$. Given $z \in \mathcal{U}$, let

$$
N(z):=\left\{k: D\left(a_{k}, r\right) \cap D(z, \delta) \neq \emptyset\right\} .
$$

Since $\left\{a_{k}\right\}$ is an $r$-lattice, we have $D(z, \delta) \subset \cup_{k \in N(z)} D\left(a_{k}, r\right)$. Thus,

$$
\mu(D(z, \delta)) \leq \sum_{k \in N(z)} \mu\left(D\left(a_{k}, r\right)\right) .
$$

Together this with (2.5) and (2.6), there exists a positive constant $C$ depending on $r$ and $\delta$ such that

$$
\widehat{\mu}_{\delta}(z) \leq C \sum_{k \in N(z)} \frac{\boldsymbol{\rho}\left(a_{k}\right)^{n+1}}{\boldsymbol{\rho}(z)^{n+1}} \widehat{\mu}_{r}\left(a_{k}\right) \leq C \sum_{k \in N(z)} \widehat{\mu}_{r}\left(a_{k}\right) .
$$

On the other hand, by Lemma 2.2. we see that $D(z, \delta)$ meets at most $N$ of the sets $D\left(a_{k}, r\right)$. Therefore,

$$
\sup _{z \in \mathcal{U}}|N(z)| \leq N<\infty
$$

where $|N(z)|$ denotes the number of elements in $N(z)$. This together with (3.2) gives

$$
\widehat{\mu}_{\delta}(z)^{p} \leq C^{p} N^{p} \sum_{k \in N(z)} \widehat{\mu}_{r}\left(a_{k}\right)^{p}
$$


for all $z \in \mathcal{U}$. Now, integrating both sides of the above against the measure $d \lambda$ and then applying Fubini's theorem, we get that there is another positive constant $C$ such that

$$
\int_{\mathcal{U}} \widehat{\mu}_{\delta}(z)^{p} \lambda(z) \leq C \int_{\mathcal{U}} \sum_{k \in N(z)} \widehat{\mu}_{r}\left(a_{k}\right)^{p} d \lambda(z)=C \sum_{k=1}^{\infty} \widehat{\mu}_{r}\left(a_{k}\right)^{p} \lambda(Q(k)),
$$

where $Q(k)=\left\{z \in \mathcal{U}: D(z, \delta) \cap D\left(a_{k}, r\right) \neq \emptyset\right\}$. Note that $Q(k) \subset D\left(a_{k}, r+\delta\right)$ and by (3.1) there exists a positive constant $C$ depending on $r+\delta$ such that $\lambda\left(D\left(a_{k}, r+\right.\right.$ $\delta)) \leq C$ for all $k \geq 1$. Thus $\lambda(Q(k)) \leq C$ for all $k \geq 1$. Combining this with the above estimate, we conclude that

$$
\int_{\mathcal{U}} \widehat{\mu}_{\delta}(z)^{p} \lambda(z) \leq C \sum_{k=1}^{\infty} \widehat{\mu}_{r}\left(a_{k}\right)^{p} .
$$

This completes the proof of the lemma.

Lemma 3.2. Suppose $\mu \geq 0, r>0$ and $0<p<\infty$. If $\widehat{\mu}_{2 r} \in L^{p}(\mathcal{U}, d \lambda)$, then for every $r$-lattice $\left\{a_{k}\right\}$ we have $\left\{\widehat{\mu}_{r}\left(a_{k}\right)\right\} \in l^{p}$.

Proof. Fix an $r$-lattice $\left\{a_{k}\right\}$. If $z \in D\left(a_{k}, r\right)$, then $D\left(a_{k}, r\right) \subset D(z, 2 r)$. By (2.5) and (2.6), there exists a positive constant $C$ depending only on $r$ such that

$$
\widehat{\mu}_{r}\left(a_{k}\right) \leq C \frac{\mu\left(D\left(a_{k}, r\right)\right)}{\boldsymbol{\rho}\left(a_{k}\right)^{n+1}} \leq C \frac{\mu(D(z, 2 r))}{\boldsymbol{\rho}(z)^{n+1}} \leq C \widehat{\mu}_{2 r}(z)
$$

for $z \in D\left(a_{k}, r\right)$. This together with (3.1) that $\lambda\left(D\left(a_{k}, r\right)\right) \leq C$ for $k \geq 1$ gives

$$
\widehat{\mu}_{r}\left(a_{k}\right)^{p} \leq C \int_{D\left(a_{k}, r\right)} \widehat{\mu}_{2 r}(z)^{p} d \lambda(z), \quad k \geq 1 .
$$

Hence,

$$
\begin{aligned}
\sum_{k=1}^{\infty} \widehat{\mu}_{r}\left(a_{k}\right)^{p} & \leq C \sum_{k=1}^{\infty} \int_{D\left(a_{k}, r\right)} \widehat{\mu}_{2 r}(z)^{p} d \lambda(z) \\
& \leq C N \int_{\mathcal{U}} \widehat{\mu}_{2 r}(z)^{p} d \lambda(z),
\end{aligned}
$$

where $N$ is as in Lemma 2.2 This completes the proof of the lemma.

As a consequence of the two lemmas above, we have the following result.

Theorem 3.3. Suppose $\mu \geq 0, r, s, \delta>0$ and $0<p<\infty$. Then the following conditions are equivalent:

(i) $\left\{\widehat{\mu}_{r}\left(a_{k}\right)\right\} \in l^{p}$ for every $r$-lattice $\left\{a_{k}\right\}$.

(ii) $\left\{\widehat{\mu}_{s}\left(a_{m}\right)\right\} \in l^{p}$ for some s-lattice $\left\{a_{m}\right\}$.

(iii) $\widehat{\mu}_{\delta} \in L^{p}(\mathcal{U}, d \lambda)$.

\section{THE CASE $p \geq 1$}

In this section we prove Theorem 1.1 in the case of $p \geq 1$. The left case $0<p<1$ will be discussed in next two sections. Before it, we shall briefly review the notion of Schatten class operators. 
For a positive compact operator $T$ on a a separable Hilbert space $H$, there exists an orthonormal set $\left\{e_{k}\right\}$ in $H$ and a sequence $\left\{\lambda_{k}\right\}$ that decreases to 0 such that

$$
T x=\sum_{k} \lambda_{k}\left\langle x, e_{k}\right\rangle e_{k}
$$

for all $x \in H$, where $\langle$,$\rangle denotes the inner product on H$. For $0<p<\infty$, we say that a positive operator $T$ belongs to the Schatten class $S_{p}(H)$ if

$$
\|T\|_{p}:=\left[\sum_{k} \lambda_{k}^{p}\right]^{1 / p}<\infty .
$$

More generally, given a compact (not necessarily positive) operator $T$ on $H$, we say that $T \in S_{p}(H)$ if the positive operator $|T|=\left(T^{*} T\right)^{1 / 2}$ belongs to $S_{p}(H)$. In this case, we define $\|T\|_{p}=\||T|\|_{p}$.

We shall recall some basic facts about $S_{p}(H)$, which we need later. Details can be found in [13].

(1) For $T \in S_{1}(H)$ and an orthonormal basis $\left\{e_{k}\right\}$ for $H$, the sum

$$
\operatorname{tr}(T)=\sum_{k}\left\langle T e_{k}, e_{k}\right\rangle
$$

is absolutely convergent and independent of the choice of $\left\{e_{k}\right\}$. This sum is called the trace of $T$.

(2) For a positive compact operator $T$ on $H$ and $0<p<\infty, T \in S_{p}(H)$ if and only if $T^{p} \in S_{1}$ and $\|T\|_{p}^{p}=\left\|T^{p}\right\|_{1}$.

(3) Suppose $T$ is a positive operator on $H$ and $x$ is a unit vector in $H$. If $p \geq 1$, then $\left\langle T^{p} x, x\right\rangle \geq\langle T x, x\rangle^{p}$.

(4) For a compact operator $T$ on $H$, if $p \geq 1$, then $T \in S_{p}(H)$ if and only if

$$
\sup \sum_{k}\left|\left\langle T e_{k}, e_{k}\right\rangle\right|^{p}<\infty
$$

where the supremum is taken over all orthonormal set $\left\{e_{k}\right\}$. Moreover, the left side of the above is the same as $\|T\|_{p}^{p}$ for $T \in S_{p}(H)$.

We will take $H=A^{2}(\mathcal{U})$ in our considerations and, in that case, hereafter, we write $S_{p}=S_{p}\left(A^{2}(\mathcal{U})\right)$.

Lemma 4.1. Let $\mu \in \mathcal{M}_{+}$. If $K(z, z) \in L^{1}(\mu)$, then $T_{\mu}$ is compact.

Proof. Note that $K(z, z)=\left\|K_{z}\right\|_{2}^{2}$, thus we have

$$
\begin{aligned}
\widetilde{\mu}(z) & =\int_{\mathcal{U}}\left\{\frac{\left|K_{z}(w)\right|}{\left\|K_{z}\right\|_{2}\left\|K_{w}\right\|_{2}}\right\}^{2} K(w, w) d \mu(w) \\
& :=\int_{\mathcal{U}} F(z, w) K(w, w) d \mu(w)
\end{aligned}
$$

On one hand, from the proof of [7, Lemma 2.12] we can see that $K_{z}\left\|K_{z}\right\|_{2}^{-1} \rightarrow 0$ uniformly on every compact subset of $\mathcal{U}$ as $z \rightarrow b \mathcal{U} \cup\{\infty\}$, where $b \mathcal{U}=\left\{z \in \mathbb{C}^{n}\right.$ : $\boldsymbol{\rho}(z)=0\}$ denotes the boundary of $\mathcal{U}$. Therefore, for every $w \in \mathcal{U}, F(z, w) \rightarrow 0$ as $z \rightarrow b \mathcal{U} \cup\{\infty\}$. On the other hand, it follows by (2.3) that $F(z, w)$ is bounded. Hence, the dominated convergence theorem allows us to take the limit inside the integral of the above and deduce that $\widetilde{\mu}$ vanishes on $b \mathcal{U} \cup\{\infty\}$. This implies by [7, Theorem 1.2] that $T_{\mu}$ is compact. 
Before proving the following lemma, we need to clarify that the well definition of $T_{f}$ with $f \in L^{p}(\mathcal{U}, d \lambda)$ for $1 \leq p<\infty$. Let $d \mu=|f| d V$, it suffices to show that

$$
\int_{\mathcal{U}} \frac{d \mu(z)}{|\boldsymbol{\rho}(\mathbf{i}, z)|^{\alpha}}<\infty
$$

for sufficiently large $\alpha$. When $1<p<\infty$, let $p^{\prime}=p /(p-1)$ be the conjugate index of $p$, by the Hölder's inequality we have

$$
\begin{aligned}
\int_{\mathcal{U}} \frac{d \mu(z)}{|\boldsymbol{\rho}(\mathbf{i}, z)|^{\alpha}} & =\int_{\mathcal{U}} \frac{|f(z)| d V(z)}{|\boldsymbol{\rho}(\mathbf{i}, z)|^{(n+1) / p+\alpha-(n+1) / p}} \\
& \leq\left[\int_{\mathcal{U}} \frac{|f(z)|^{p}}{|\boldsymbol{\rho}(\mathbf{i}, z)|^{n+1}} d V(z)\right]^{1 / p}\left[\int_{\mathcal{U}} \frac{d V(z)}{|\boldsymbol{\rho}(\mathbf{i}, z)|^{(\alpha-(n+1) / p) p^{\prime}}}\right]^{1 / p^{\prime}} \\
& \leq C\left[\int_{\mathcal{U}}|f(z)|^{p} K(z, z) d V(z)\right]^{1 / p}\left[\int_{\mathcal{U}} \frac{d V(z)}{|\boldsymbol{\rho}(\mathbf{i}, z)|^{(\alpha-(n+1) / p) p^{\prime}}}\right]^{1 / p^{\prime}}
\end{aligned}
$$

where the last inequality follows from (2.3). By (2.2), the second integral of above is finite if and only if $(\alpha-(n+1) / p) p^{\prime}>n+1$, which is equivalent to $\alpha>n+1$. Hence, in the case of $1<p<\infty$, (4.1) holds for $\alpha>n+1$. To prove the remained case $p=1$, by letting $\alpha=n+1$ and using (2.3) again, we obtain

$$
\int_{\mathcal{U}} \frac{d \mu(z)}{|\boldsymbol{\rho}(\mathbf{i}, z)|^{\alpha}}=\int_{\mathcal{U}} \frac{|f(z)|}{|\boldsymbol{\rho}(\mathbf{i}, z)|^{n+1}} d V(z) \leq C \int_{\mathcal{U}}|f(z)| K(z, z) d V(z),
$$

as desired.

Lemma 4.2. Let $1 \leq p<\infty$. If $f \in L^{p}(\mathcal{U}, d \lambda)$, then $T_{f} \in S_{p}$.

Proof. By the Riesz-Thorin type interpolation theorem for Schatten classes (see [13. Section 2.2] for example), it suffices to show that the map $f \mapsto T_{f}$ is bounded from $L^{1}(\mathcal{U}, d \lambda)$ into $S_{1}$ (the case $p=\infty$ is trivial). So, assume that $f \in L^{1}(\mathcal{U}, d \lambda)$. Then $K(z, z) \in L^{1}(\mu)$ for $d \mu=|f| d V$. According to Lemma 4.1 we get that $T_{\mu}$ which is $T_{|f|}$ is compact, and so is $T_{f}$. To estimate the trace of $T_{f}$, let $\left\{e_{k}\right\}$ be any orthonormal set in $A^{2}(\mathcal{U})$. Note that

$$
\left\langle T_{f} e_{k}, e_{k}\right\rangle=\int_{\mathcal{U}} f\left|e_{k}\right|^{2} d V
$$

for each $k$. Also, recall that $\sum\left|e_{k}(z)\right|^{2} \leq K(z, z)$. Therefore, we have

$$
\sum_{k}\left|\left\langle T_{f} e_{k}, e_{k}\right\rangle\right| \leq \int_{\mathcal{U}}|f| \sum_{k}\left|e_{k}\right|^{2} d V \leq \int_{\mathcal{U}}|f| d \lambda .
$$

It follows that $T_{f} \in S_{1}$ and

$$
\left\|T_{f}\right\|_{1} \leq \int_{\mathcal{U}}|f| d \lambda
$$

This completes the proof of the lemma. by

For a bounded linear operator on $A^{2}(\mathcal{U})$, the Berezin transform $\widetilde{T}$ of $T$ is defined

$$
\widetilde{T}(z)=\left\langle T k_{z}, k_{z}\right\rangle, \quad z \in \mathcal{U} .
$$

For $\mu \in \mathcal{M}_{+}$, if $T_{\mu}$ is bounded on $A^{2}(\mathcal{U})$, then $\widetilde{T_{\mu}}=\widetilde{\mu}$. 
Lemma 4.3. If $T$ is a positive operator on $A^{2}(\mathcal{U})$, then $T \in S_{1}$ if and only if $\widetilde{T} \in L^{1}(\mathcal{U}, \lambda)$. Moreover,

$$
\operatorname{tr}(T)=\int_{\mathcal{U}} \widetilde{T}(z) d \lambda(z) .
$$

Proof. The proof is similar to that of [13, Theorem 6.4], so we omit the details here.

Lemma 4.4. Suppose $r>0$ and $\mu \in \mathcal{M}_{+}$. If $T_{\widehat{\mu}_{r}}$ is bounded on $A^{2}(\mathcal{U})$, then so is $T_{\mu}$ with $T_{\mu} \leq C_{r} T_{\widehat{\mu}_{r}}$ for a constant $C_{r}>0$.

Proof. Given $f \in A^{2}(\mathcal{U})$, Fubini's theorem gives

$$
\begin{aligned}
\left\langle T_{\widehat{\mu}_{r}} f, f\right\rangle & =\int_{\mathcal{U}}|f(z)|^{2} \widehat{\mu}_{r} d V(z) \\
& =\int_{\mathcal{U}}|f(z)|^{2} \frac{\mu(D(z, r))}{|D(z, r)|} d V(z) \\
& =\int_{\mathcal{U}} \frac{|f(z)|^{2}}{|D(z, r)|} d V(z) \int_{D(z, r)} d \mu(w) \\
& =\int_{\mathcal{U}} d \mu(w) \int_{D(w, r)} \frac{|f(z)|^{2}}{|D(z, r)|} d V(z) .
\end{aligned}
$$

By (2.4) and (2.6), we can see that $|D(z, r)|$ is comparable to $|D(w, r)|$ for all $w \in \mathcal{U}$ and $z \in D(w, r)$. This together with Lemma 2.7 implies that there exists a positive constant $C$ such that

$$
|f(w)|^{2} \leq C \int_{D(w, r)} \frac{|f(z)|^{2}}{|D(z, r)|} d V(z)
$$

for all $w \in \mathcal{U}$. It follows that

$$
\left\langle T_{\mu} f, f\right\rangle=\int_{\mathcal{U}}|f(w)|^{2} d \mu(w) \leq C\left\langle T_{\widehat{\mu}_{r}} f, f\right\rangle
$$

for all $f \in A^{2}(\mathcal{U})$, completing the proof of the lemma.

Theorem 4.5. Suppose $p \geq 1, r>0$ and $\mu \in \mathcal{M}_{+}$. Then the following conditions are equivalent:

(i) $T_{\mu} \in S_{p}$.

(ii) $\widetilde{\mu} \in L^{p}(\mathcal{U}, d \lambda)$.

(iii) $\widehat{\mu}_{r} \in L^{p}(\mathcal{U}, d \lambda)$.

(iv) $\left\{\widehat{\mu}_{r}\left(a_{k}\right)\right\} \in l^{p}$ for every $r$-lattice $\left\{a_{k}\right\}$.

(v) $\left\{\widehat{\mu}_{r}\left(a_{k}\right)\right\} \in l^{p}$ for some r-lattice $\left\{a_{k}\right\}$

Proof. (i) $\Rightarrow$ (ii). Assume $T_{\mu} \in S_{p}$. Then we have $T_{\mu}^{p} \in S_{1}$ and $\widetilde{T_{\mu}^{p}} \geq\left(\widetilde{T_{\mu}}\right)^{p}$ (see [13, Proposition 1.31] for example). Combining the fact that $\widetilde{T_{\mu}}=\widetilde{\mu}$ with (4.2), we have

$$
\int_{\mathcal{U}}(\widetilde{\mu})^{p} d \lambda=\int_{\mathcal{U}}\left(\widetilde{T_{\mu}}\right)^{p} d \lambda \leq \int_{\mathcal{U}} \widetilde{T_{\mu}^{p}} d \lambda=\operatorname{tr}\left(T_{\mu}^{p}\right)<\infty .
$$

(ii) $\Rightarrow$ (iii). This easily follows by

$$
\widehat{\mu}_{r}(z)=C \boldsymbol{\rho}(z)^{-n-1} \int_{D(z, r)} d \mu(w) \leq C \int_{D(z, r)}\left|k_{z}(w)\right|^{2} d \mu(w) \leq C \widetilde{\mu}(z),
$$


where the expression is due to (2.5) and (2.6).

(iii) $\Rightarrow$ (i). Assume $\widehat{\mu}_{r} \in L^{p}(\mathcal{U}, d \lambda)$. Thus by Lemma 4.2, $T_{\widehat{\mu}_{r}} \in S_{p}$. Then it immediately follows from Lemma 4.4 that $T_{\mu} \in S_{p}$.

That implication (iii) $\Leftrightarrow$ (iv) $\Leftrightarrow$ (v) follows immediately from Theorem 3.3. The proof of the theorem is complete.

\section{The CASE $0<p<1$ : PART I}

In this section we describe our main result except the integral properties of Berezin transform $\widetilde{\mu}$ in the range of $0<p<1$, where the methods involved are adapted from [11. The key of the section is of characterization of the membership of $T_{\mu}$ in $S_{p}$ in terms of the averaging function $\widehat{\mu}_{r}$. We begin with the following three lemmas, which could be tracked in 13 .

Lemma 5.1. Suppose $A$ is a bounded surjective operator on $H$ and $T$ is any bounded linear operator on $H$. Then $T \in S_{p}$ if and only if $A^{*} T A \in S_{p}$.

Lemma 5.2. Suppose $0<p \leq 2$ and $T$ is a compact operator on $H$. Then

$$
\|T\|_{p}^{p} \leq \sum_{i=1}^{\infty} \sum_{j=1}^{\infty}\left|\left\langle T e_{i}, e_{j}\right\rangle\right|^{p}
$$

for any orthonormal basis $\left\{e_{k}\right\}$ of $H$.

Lemma 5.3. Suppose $T$ is a positive compact operator on $H$ and $\left\{e_{k}\right\}$ is any orthonormal basis of $H$. If $0<p \leq 1$ and

$$
\sum_{k=1}^{\infty}\left\langle T e_{k}, e_{k}\right\rangle^{p}<\infty
$$

then $T$ belongs to $S_{p}$.

Lemma 5.4. Suppose $0<p<1, r>0$ and $\mu \in \mathcal{M}_{+}$. If there exists an $r$-lattice $\left\{a_{k}\right\}$ such that $\left\{\widehat{\mu}_{r}\left(a_{k}\right)\right\} \in l^{p}$, then $T_{\mu} \in S_{p}$.

Proof. Suppose $\left\{a_{k}\right\}$ is an $r$-lattice such that $\left\{\widehat{\mu}_{r}\left(a_{k}\right)\right\} \in l^{p}$. First note from [7] that $T_{\mu}$ is compact on $A^{2}(\mathcal{U})$. We want to show that $T_{\mu}$ is in $S_{p}$. To this end, fix a sufficiently large number $b$ and by [3, Theorem 2] (the atomic decomposition of functions in Bergman spaces on symmetric Siegel domains of type two), we may assume that $A^{2}(\mathcal{U})$ consists exactly of functions of the form

$$
f(z)=\sum_{k=1}^{\infty} c_{k} h_{k}(z),
$$

where $\left\{c_{k}\right\} \in l^{2}$,

$$
h_{k}(z)=\frac{\boldsymbol{\rho}\left(a_{k}\right)^{b-(n+1) / 2}}{\boldsymbol{\rho}\left(z, a_{k}\right)^{b}},
$$

and

$$
\int_{\mathcal{U}}|f(z)|^{2} d V(z) \leq C \sum_{k=1}^{\infty}\left|c_{k}\right|^{2}
$$

for some positive constant $C$ independent of $\left\{c_{k}\right\}$. 
Fix an orthonormal basis $\left\{e_{k}\right\}$ for $A^{2}(\mathcal{U})$ and define an operator $A$ on $A^{2}(\mathcal{U})$ by

$$
A\left(\sum_{k=1}^{\infty} c_{k} e_{k}\right)=\sum_{k=1}^{\infty} c_{k} h_{k}
$$

By the statements of above paragraph, we can see that $A$ is a bounded surjective operator on $A^{2}(\mathcal{U})$. Applying Lemma 5.1, the Toeplitz operator $T_{\mu}$ will be in $S_{p}$ if we can show that the operator $T=A^{*} T_{\mu} A$ belongs to $S_{p}$. To show that $T \in S_{p}$, according to Lemma 5.3 we just need to verify that

$$
M=\sum_{k=1}^{\infty}\left\langle T e_{k}, e_{k}\right\rangle^{p}<\infty
$$

First we note that

$$
\left\langle T e_{k}, e_{k}\right\rangle=\left\langle T_{\mu} h_{k}, h_{k}\right\rangle=\int_{\mathcal{U}}\left|h_{k}(z)\right|^{2} d \mu(z) \leq \sum_{j=1}^{\infty} \int_{D\left(a_{j}, r\right)}\left|h_{k}(z)\right|^{2} d \mu(z) .
$$

By (2.6) and (2.5), there is a positive constant $C$ such that

$$
\left\langle T e_{k}, e_{k}\right\rangle \leq C \sum_{j=1}^{\infty}\left|h_{k}\left(a_{j}\right)\right|^{2} \boldsymbol{\rho}\left(a_{j}\right)^{n+1} \widehat{\mu}_{r}\left(a_{j}\right) .
$$

Since $0<p<1$, an application of Höder's inequality gives

$$
\left\langle T e_{k}, e_{k}\right\rangle^{p} \leq C \sum_{j=1}^{\infty}\left|h_{k}\left(a_{j}\right)\right|^{2 p} \boldsymbol{\rho}\left(a_{j}\right)^{p(n+1)} \widehat{\mu}_{r}\left(a_{j}\right)^{p} .
$$

Thus by Fubini's theorem, we obtain

$$
M \leq C \sum_{j=1}^{\infty} \widehat{\mu}_{r}\left(a_{j}\right)^{p} \boldsymbol{\rho}\left(a_{j}\right)^{p(n+1)} \sum_{k=1}^{\infty}\left|h_{k}\left(a_{j}\right)\right|^{2 p} .
$$

For each $j$ we consider the sum

$$
M_{j}=\sum_{k=1}^{\infty}\left|h_{k}\left(a_{j}\right)\right|^{2 p}=\sum_{k=1}^{\infty} \frac{\boldsymbol{\rho}\left(a_{k}\right)^{p(2 b-n-1)}}{\left|\boldsymbol{\rho}\left(a_{j}, a_{k}\right)\right|^{2 p b}} .
$$

By Lemma 2.7 there exists a positive constant $C$ such that

$$
\frac{1}{\left|\boldsymbol{\rho}\left(a_{j}, a_{k}\right)\right|^{2 p b}} \leq \frac{C}{\boldsymbol{\rho}\left(a_{k}\right)^{n+1}} \int_{D\left(a_{k}, r\right)} \frac{d V(z)}{\left|\boldsymbol{\rho}\left(a_{j}, z\right)\right|^{2 p b}}
$$

for all $j$ and all $k$. Since $\boldsymbol{\rho}\left(a_{k}\right)$ is comparable to $\boldsymbol{\rho}(z)$ for $z \in D\left(a_{k}, r\right)$, we have

$$
\begin{aligned}
M_{j} & \leq C \sum_{k=1}^{\infty} \int_{D\left(a_{k}, r\right)} \frac{\boldsymbol{\rho}(z)^{p(2 b-n-1)-n-1}}{\left|\boldsymbol{\rho}\left(a_{j}, z\right)\right|^{2 p b}} d V(z) \\
& \leq C N \int_{\mathcal{U}} \frac{\boldsymbol{\rho}(z)^{p(2 b-n-1)-n-1}}{\left|\boldsymbol{\rho}\left(a_{j}, z\right)\right|^{2 p b}} d V(z),
\end{aligned}
$$

where $N$ is as in Lemma 2.2. We can assume that $b$ is large enough so that $p(2 b-n-1)>n$, then applying (2.2), there exists a positive constant $C$ such that

$$
M_{j} \leq C \boldsymbol{\rho}\left(a_{j}\right)^{-p(n+1)}
$$


for all $j$. Hence, it follows that

$$
M \leq C \sum_{j=1}^{\infty} \widehat{\mu}_{r}\left(a_{j}\right)^{p}<\infty .
$$

This completes the proof of the lemma.

Lemma 5.5. Suppose $0<p<1, r>0$ and $\mu \in \mathcal{M}_{+}$. If $T_{\mu} \in S_{p}$ and $\left\{a_{k}\right\}$ is an $r$-lattice, then $\left\{\widehat{\mu}_{r}\left(a_{k}\right)\right\} \in l^{p}$.

Proof. Fix a sufficiently large positive number $R$. Lemma 2.2 tells us that there is a decomposition of $\left\{a_{k}\right\}$ into $m$ subsequences $\left\{\Gamma_{i}\right\}$ such that for every pair $u, v \in \Gamma_{i}$ with $u \neq v, \beta(u, v)>R$. Let $\left\{\zeta_{j}\right\}$ be some $\Gamma_{i}$ and define a measure $\nu$ as follows:

$$
d \nu(z)=\sum_{k=1}^{\infty} \chi_{k}(z) d \mu(z),
$$

where $\chi_{k}$ is the characteristic function of $D\left(\zeta_{k}, r\right)$. Assume that $R>2 r$, then the Bergman metric balls $\left\{D\left(\zeta_{k}, r\right)\right\}$ are disjoint. Also, note that $0 \leq \nu \leq \mu$, we have $\nu \in \mathcal{M}_{+}$and $T_{\nu} \in S_{p}$ with $\left\|T_{\nu}\right\|_{p} \leq\left\|T_{\mu}\right\|_{p}$.

Fix an orthonormal basis $\left\{e_{k}\right\}$ for $A^{2}(\mathcal{U})$. Similar to (5.1), we define an auxiliary bounded operator

$$
A\left(\sum_{k=1}^{\infty} c_{k} e_{k}\right)=\sum_{k=1}^{\infty} c_{k} h_{k}
$$

where

$$
h_{k}(z)=\frac{\boldsymbol{\rho}\left(\zeta_{k}\right)^{b-(n+1) / 2}}{\boldsymbol{\rho}\left(z, \zeta_{k}\right)^{b}}
$$

and $b$ is sufficiently large.

Put $T=A^{*} T_{\nu} A$. Since $A$ is bounded and $T_{\nu} \in S_{p}$, we have $T \in S_{p}$ with $\|T\|_{p} \leq\|A\|^{2}\left\|T_{\nu}\right\|_{p}$. Hence, there exists a positive constant $C$ such that

$$
\|T\|_{p}^{p} \leq C\left\|T_{\mu}\right\|_{p}^{p} .
$$

We split the operator $T$ as $T=D+E$, where $D$ is the diagonal operator defined by

$$
D f=\sum_{k=1}^{\infty}\left\langle T e_{k}, e_{k}\right\rangle\left\langle f, e_{k}\right\rangle e_{k}, \quad f \in A^{2}(\mathcal{U})
$$

and $E=T-D$.

Note that $D$ is compact and positive, we have

$$
\begin{aligned}
\|D\|_{p}^{p} & =\sum_{k=1}^{\infty}\left\langle T e_{k}, e_{k}\right\rangle^{p}=\sum_{k=1}^{\infty}\left\langle T_{\nu} h_{k}, h_{k}\right\rangle^{p} \\
& =\sum_{k=1}^{\infty}\left[\int_{\mathcal{U}}\left|h_{k}(z)\right|^{2} d \nu(z)\right]^{p} \geq \sum_{k=1}^{\infty}\left[\int_{D\left(\zeta_{k}, r\right)}\left|h_{k}(z)\right|^{2} d \nu(z)\right]^{p} \\
& \geq C \sum_{k=1}^{\infty} \widehat{\nu}_{r}\left(\zeta_{k}\right)^{p}
\end{aligned}
$$


where the last inequality follows by (2.5) and (2.6). Since $\nu=\mu$ on each $D\left(\zeta_{k}, r\right)$, we obtain

$$
\|D\|_{p}^{p} \geq C_{1} \sum_{k=1}^{\infty} \widehat{\mu}_{r}\left(\zeta_{k}\right)^{p} .
$$

On the other hand, by Lemma 5.2 we have

$$
\begin{aligned}
\|E\|_{p}^{p} & \leq \sum_{i=1}^{\infty} \sum_{j=1}^{\infty}\left|\left\langle E e_{i}, e_{j}\right\rangle\right|^{p}=\sum_{j \neq k}\left|\left\langle T_{\nu} h_{j}, h_{k}\right\rangle\right|^{p} \\
& =\sum_{j \neq k}\left|\int_{\mathcal{U}} h_{j}(z) \overline{h_{k}(z)} d \nu(z)\right|^{p} \\
& \leq \sum_{j \neq k}\left[\sum_{i=1}^{\infty} \int_{D\left(\zeta_{i}, r\right)}\left|h_{j}(z) h_{k}(z)\right| d \mu(z)\right]^{p} .
\end{aligned}
$$

Again by (2.5) and (2.6), there exists a positive $C$ such that

$$
\int_{D\left(\zeta_{i}, r\right)}\left|h_{j}(z) h_{k}(z)\right| d \mu(z) \leq C \boldsymbol{\rho}\left(\zeta_{i}\right)^{n+1}\left|h_{j}\left(\zeta_{i}\right) h_{k}\left(\zeta_{i}\right)\right| \widehat{\mu}_{r}\left(\zeta_{i}\right)
$$

for all $i$. Since $0<p<1$, an application of Hölder's inequality gives

$$
\|E\|_{p}^{p} \leq C \sum_{j \neq k} \sum_{i=1}^{\infty} \boldsymbol{\rho}\left(\zeta_{i}\right)^{p(n+1)}\left|h_{j}\left(\zeta_{i}\right) h_{k}\left(\zeta_{i}\right)\right|^{p} \widehat{\mu}_{r}\left(\zeta_{i}\right)^{p} .
$$

Then using Fubini's theorem, we obtain

$$
\|E\|_{p}^{p} \leq C \sum_{i=1}^{\infty} \boldsymbol{\rho}\left(\zeta_{i}\right)^{p(n+1)} \widehat{\mu}_{r}\left(\zeta_{i}\right)^{p} I_{i}
$$

where

$$
I_{i}=\sum_{j \neq k}\left|h_{j}\left(\zeta_{i}\right) h_{k}\left(\zeta_{i}\right)\right|^{p} .
$$

Since every $\left|h_{j}\left(\zeta_{i}\right)\right|^{p}$ is comparable to

$$
\int_{D\left(\zeta_{j}, r\right)} \frac{\boldsymbol{\rho}(z)^{p b-p(n+1) / 2}}{\left|\boldsymbol{\rho}\left(\zeta_{i}, z\right)\right|^{p b}} d \lambda(z)
$$

by (2.4) and (2.6), and since $\Omega=\bigcup_{j \neq k} D\left(\zeta_{j}, r\right) \times D\left(\zeta_{k}, r\right)$ is a disjoint union, we can find a positive constant $C$ such that

$$
I_{i} \leq C \iint_{\Omega} \frac{[\boldsymbol{\rho}(z) \boldsymbol{\rho}(w)]^{p b-p(n+1) / 2}}{\left[\mid \boldsymbol{\rho}\left(\zeta_{i}, z\right) \| \boldsymbol{\rho}\left(\zeta_{i}, w\right)\right]^{p b}} d \lambda(z) d \lambda(w) .
$$

By assumption, we have

$$
\Omega \subset G_{R}=\{(z, w) \in \mathcal{U} \times \mathcal{U}: \beta(z, w) \geq R-2 r\} .
$$

Hence,

$$
I_{i} \leq C \iint_{G_{R}} \frac{[\boldsymbol{\rho}(z) \boldsymbol{\rho}(w)]^{p b-p(n+1) / 2}}{\left[\mid \boldsymbol{\rho}\left(\zeta_{i}, z\right) \| \boldsymbol{\rho}\left(\zeta_{i}, w\right)\right]^{p b}} d \lambda(z) d \lambda(w) .
$$

Making the change of $z=\sigma_{\zeta_{i}}^{-1}(u)$ and $w=\sigma_{\zeta_{i}}^{-1}(v)$, by (2.1) we obtain

$$
I_{i} \leq C \boldsymbol{\rho}\left(\zeta_{i}\right)^{-p(n+1)} \iint_{G_{R}} \frac{[\boldsymbol{\rho}(u) \boldsymbol{\rho}(v)]^{p b-p(n+1) / 2}}{[\mid \boldsymbol{\rho}(\mathbf{i}, u) \| \boldsymbol{\rho}(\mathbf{i}, v)]^{p b}} d \lambda(u) d \lambda(v) .
$$


Therefore, there is a positive constant $C_{2}$ such that

$$
\|E\|_{p}^{p} \leq C_{2} C_{R} \sum_{i=1}^{\infty} \widehat{\mu}_{r}\left(\zeta_{i}\right)^{p}
$$

where

$$
C_{R}=\iint_{G_{R}} \frac{[\boldsymbol{\rho}(u) \boldsymbol{\rho}(v)]^{p b-p(n+1) / 2-n-1}}{[\mid \boldsymbol{\rho}(\mathbf{i}, u) \| \boldsymbol{\rho}(\mathbf{i}, v)]^{p b}} d V(u) d V(v) .
$$

We can assume that $b$ is large enough so that $p b>p(n+1) / 2+n$, thus it follows by (2.2) that

$$
\int_{\mathcal{U}} \frac{\boldsymbol{\rho}(u)^{p b-p(n+1) / 2-n-1}}{|\boldsymbol{\rho}(\mathbf{i}, u)|^{p b}} d V(u)<\infty .
$$

Consequently, it follows that $C_{R} \rightarrow 0$ as $R \rightarrow \infty$.

Finally, by the triangle inequality, we know that

$$
\|T\|_{p}^{p} \geq\|D\|_{p}^{p}-\|T\|_{p}^{p}
$$

In view of (5.3) and (5.4), we have

$$
\|T\|_{p}^{p} \geq\left(C_{1}-C_{2} C_{R}\right) \sum_{i=1}^{\infty} \widehat{\mu}_{r}\left(\zeta_{i}\right)^{p} .
$$

It is clear that $C_{1}$ and $C_{2}$ are independent of $R$. We can chose $R$ large enough so that $C_{1}-C_{2} C_{R}>0$. Together with (5.2), it follows that there exists a positive constant $C$ such that

$$
\sum_{i=1}^{\infty} \widehat{\mu}_{r}\left(\zeta_{i}\right)^{p} \leq C\left\|T_{\mu}\right\|_{p}^{p} .
$$

Since this holds for each one of the $m$ subsequences of $\left\{a_{k}\right\}$, it follows that

$$
\sum_{k=1}^{\infty} \widehat{\mu}_{r}\left(a_{k}\right)^{p} \leq C m\left\|T_{\mu}\right\|_{p}^{p}
$$

This completes the proof of the lemma.

As a consequence of the two lemmas above, we see that $T_{\mu} \in S_{p}$ if and only if $\left\{\widehat{\mu}_{r}\left(a_{k}\right)\right\} \in l^{p}$ for some (every) $r$-lattice $\left\{a_{k}\right\}$. Associating with Theorem 3.3, we conclude the main result of the section.

Theorem 5.6. Suppose $0<p<1, r>0$ and $\mu \in \mathcal{M}_{+}$. Then the following conditions are equivalent:

(i) $T_{\mu} \in S_{p}$.

(ii) $\widehat{\mu}_{r} \in L^{p}(\mathcal{U}, d \lambda)$.

(iii) $\left\{\widehat{\mu}_{r}\left(a_{k}\right)\right\} \in l^{p}$ for every $r$-lattice $\left\{a_{k}\right\}$.

(iii) $\left\{\widehat{\mu}_{r}\left(a_{k}\right)\right\} \in l^{p}$ for some r-lattice $\left\{a_{k}\right\}$.

\section{The Case $0<p<1$ : Part II}

In this section we characterize the membership of $T_{\mu}$ in $S_{p}$ by integral properties of the Berezin transform $\widetilde{\mu}$ in the range of $0<p<1$. It turns out that this can not be done for the full range. We begin the section with showing the obstruction. 
If $\mu$ is any positive Borel measure on $\mathcal{U}$, a use of (2.6) shows that

$$
\begin{aligned}
\widetilde{\mu}(z) & =\frac{n !}{4 \pi^{n}} \int_{\mathcal{U}} \frac{\boldsymbol{\rho}(z)^{n+1}}{|\boldsymbol{\rho}(z, w)|^{2(n+1)}} d \mu(w) \\
& \geq \frac{n !}{4 \pi^{n}} \int_{D(\mathbf{i}, r)} \frac{\boldsymbol{\rho}(z)^{n+1}}{|\boldsymbol{\rho}(z, w)|^{2(n+1)}} d \mu(w) \\
& \geq C \mu(D(\mathbf{i}, r)) \frac{\boldsymbol{\rho}(z)^{n+1}}{|\boldsymbol{\rho}(z, \mathbf{i})|^{2(n+1)}} \\
& \geq C_{1} \frac{\boldsymbol{\rho}(z)^{n+1}}{|\boldsymbol{\rho}(z, \mathbf{i})|^{2(n+1)}},
\end{aligned}
$$

where $C$ and $C_{1}$ are positive constants independent of $z$. Thus, an application of (2.2) implies that

$$
\int_{\mathcal{U}} \widetilde{\mu}(z)^{p} d \lambda(z) \geq C_{1}^{p} \int_{\mathcal{U}} \frac{\boldsymbol{\rho}(z)^{p(n+1)-n-1}}{|\boldsymbol{\rho}(z, \mathbf{i})|^{2 p(n+1)}} d V(z)=\infty
$$

whenever $p(n+1) \leq n$. Therefore, in the range $0<p \leq n /(n+1)$, it is not possible to characterize the membership of $T_{\mu}$ in $S_{p}$ in terms of the $L^{p}(\mathcal{U}, d \lambda)$-properties of $\widetilde{\mu}$. The following result shows that this is the only obstruction.

Theorem 6.1. Suppose $\mu \in \mathcal{M}_{+}$and

$$
n /(n+1)<p<1 \text {. }
$$

Then $T_{\mu} \in S_{p}$ if and only if $\widetilde{\mu} \in L^{p}(\mathcal{U}, d \lambda)$.

Proof. In view of (4.3), the condition $\widetilde{\mu} \in L^{p}(\mathcal{U}, d \lambda)$ implies that $\widehat{\mu}_{r} \in L^{p}(\mathcal{U}, d \lambda)$, which, by Theorem [5.6] implies that $T_{\mu} \in S_{p}$.

Next we assume that $T_{\mu} \in S_{p}$. Given an $r$-lattice $\left\{a_{k}\right\}$, according to Theorem 5.6. we have $\left\{\widehat{\mu}_{r}\left(a_{k}\right)\right\} \in l^{p}$. To show that $\widetilde{\mu} \in L^{p}(\mathcal{U}, d \lambda)$, it suffices to prove that the $L^{p}(\mathcal{U}, d \lambda)$-norm of $\widetilde{\mu}$ is dominated by a constant multiple of the $l^{p}$-norm of $\left\{\widehat{\mu}_{r}\left(a_{k}\right)\right\}$. By (2.6) and (2.5), there is positive constant $C$ such that

$$
\begin{aligned}
\widetilde{\mu}(z) & =\frac{n !}{4 \pi^{n}} \int_{\mathcal{U}} \frac{\boldsymbol{\rho}(z)^{n+1}}{|\boldsymbol{\rho}(z, w)|^{2(n+1)}} d \mu(w) \\
& \leq \frac{n !}{4 \pi^{n}} \sum_{k=1}^{\infty} \int_{D\left(a_{k}, r\right)} \frac{\boldsymbol{\rho}(z)^{n+1}}{|\boldsymbol{\rho}(z, w)|^{2(n+1)}} d \mu(w) \\
& \leq C \sum_{k=1}^{\infty} \frac{\boldsymbol{\rho}(z)^{n+1}}{\left|\boldsymbol{\rho}\left(z, a_{k}\right)\right|^{2(n+1)}} \mu\left(D\left(a_{k}, r\right)\right) \\
& \leq C \sum_{k=1}^{\infty} \frac{\boldsymbol{\rho}(z)^{n+1}}{\left|\boldsymbol{\rho}\left(z, a_{k}\right)\right|^{2(n+1)}} \boldsymbol{\rho}\left(a_{k}\right)^{n+1} \widehat{\mu}_{r}\left(a_{k}\right) .
\end{aligned}
$$

An application of Hölder's inequality leads to

$$
\int_{\mathcal{U}} \widetilde{\mu}(z)^{p} d \lambda(z) \leq C \sum_{k=1}^{\infty} \boldsymbol{\rho}\left(a_{k}\right)^{p(n+1)} \widehat{\mu}_{r}\left(a_{k}\right)^{p} \int_{\mathcal{U}} \frac{\boldsymbol{\rho}(z)^{p(n+1)-n-1}}{\left|\boldsymbol{\rho}\left(z, a_{k}\right)\right|^{2 p(n+1)}} d V(z) .
$$

Applying (2.2), the integrability of right hand side of above inequality is guaranteed by the assumption that $p(n+1)>n$. Therefore, it follows that there is another 
positive constant $C$ such that

$$
\int_{\mathcal{U}} \widetilde{\mu}(z)^{p} d \lambda(z) \leq C \sum_{k=1}^{\infty} \widehat{\mu}_{r}\left(a_{k}\right)^{p} .
$$

This completes the proof of the theorem.

Now, a combination of Theorem 4.5. Theorem 5.6 and Theorem 6.1 gives our main result which was stated as Theorem 1.1 in the introduction.

Remark. At the end of the paper, it should be pointed out that the methods involved in this paper are also applicable to the weighted cases.

\section{REFERENCES}

[1] B. Choe, H. Koo and H. Yi, Positive Toeplitz operators between the harmonic Bergman spaces, Potential Anal. 17 (2002), 307-335.

[2] B. Choe, H. Koo and Y. Lee, Positive Schatten(-Herz) class Toeplitz operators on the halfspace, Potential Anal. 27 (2007), 73-100.

[3] R.R. Coifman and R. Rochberg, Representation theorems for holomorphic and harmonic functions in $L^{p}$, Astérisque 77 (1980), 11-66.

[4] S. G. Gindikin, Analysis in homogeneous domains, Russian Math. Surveys 19(4) (1964), 1-89.

[5] S. G. Krantz, Function theory of several complex variables. Reprint of the 1992 edition. AMS Chelsea Publishing, Providence, RI, 2001.

[6] C. Liu, Y. Liu, P. Hu and L. Zhou, Two classes of integral operators over the Siegel upper half-space, Complex Anal. Oper. Theory (2019), no.3, 685-701.

[7] C. Liu and J. Si, Positive Toeplitz operators on the Bergman spaces of the Siegel upper half-space, Commun. Math. Stat. 8 (2020), 113-134.

[8] D. H. Luecking, Trace ideal criteria for Toeplitz operators, J. Funct. Anal. 73 (1987), 345-368.

[9] E. M. Stein, Harmonic analysis: real-variable methods, orthogonality, and oscillatory integrals. Princeton University Press, Princeton, NJ, 1993.

[10] K. Zhu, Positive Toeplitz operators on weighted Bergman spaces of bounded symmetric domains, J. Operator. Theory 20 (1988), 329-357.

[11] K. Zhu, Schatten class Toeplitz operators on weighted Bergman spaces if the unit ball, New York J. Math. 13 (2007), 299-316.

[12] K. Zhu, Spaces of Holomorphic Functions in the Unit Ball, Graduate Texts in Math., vol. 226, Springer, New York 2005.

[13] K. Zhu, Operator theory in function spaces. Second edition. Mathematical Surveys and Monographs, 138. American Mathematical Society, Providence, RI, 2007.

E-mail address: sijiajia@mail.ustc.edu.cn

School of Science, Hainan University, Haikou, Hainan 570228, Peoples Republic of CHina. 